\title{
Theoretical Study and Experimental Realization of a Low-Loss Metamaterial Operating at the Millimeter-Wave Regime: Demonstrations of Flat- and Prism-Shaped Samples
}

\author{
Kamil Boratay Alici and Ekmel Ozbay, Member, IEEE
}

\begin{abstract}
We designed a low-loss double-negative composite metamaterial that operates at the millimeter-wave regime. A negative passband with a peak transmission value of $-2.7 \mathrm{~dB}$ was obtained experimentally at $100 \mathrm{GHz}$. We performed transmissionbased qualitative effective medium theory analysis numerically and experimentally to prove the double-negative nature of the metamaterial. These results were supported by the standard retrieval analysis method and the study was extended by reporting the fractional bandwidth and loss of the metamaterial as the number of layers in the propagation direction increased. We numerically calculated 2-D field map and experimentally confirmed far-field radiation response of horn antenna and metamaterial lens composite. Finally, we demonstrated that the effective index of the metamaterial was negative by performing far-field angular scanning measurements for a metamaterial prism. We simulated the prism by using the Drude-Lorentz model and obtained the scattered field map in two dimensions at millimeter-wavelengths.
\end{abstract}

Index Terms-Metamaterials, metaprism, millimeter wave, negative refraction.

\section{INTRODUCTION}

$\mathbf{M}$ OST solid materials are in crystalline form, i.e., on the microscopic level the ions are arranged in a periodic array [1]. In the presence of an applied electromagnetic field, the materials' linear response is described by (electric) permittivity $(\varepsilon)$ and (magnetic) permeability $(\mu)$ parameters. By introducing an artificial periodic array wherein repeated elements of the so-called metamaterial are arranged, we can obtain a medium with a controllable linear response at any desired narrow frequency band up to ultraviolet. The unit cells of metamaterials are commonly composed of metallic-dielectric structures of several shapes [2]-[4]. Recently, ferroelectric and ferromagnetic materials were also utilized as constituting elements leading to

Manuscript received June 3, 2009; revised July 26, 2009. First published October 23, 2009; current version published April 7, 2010. This work was supported in part by the European Union projects EU-METAMORPHOSE, EU-PHOREMOST, EU-PHOME, and EU-ECONAM, and TUBITAK under the Project 105E066, Project 105A005, Project 106E198, and Project 106A017, and in part by the Turkish Academy of Sciences.

K. B. Alici is with the Nanotechnology Research Center, Bilkent University, Ankara 06800, Turkey, and also with the Physics Department, Bilkent University, Ankara 06800, Turkey (e-mail: bora@fen.bilkent.edu.tr).

E. Ozbay is with the Nanotechnology Research Center, Bilkent University, Ankara 06800, Turkey, and also with the Physics Department, Bilkent University, Ankara 06800, Turkey. He is also with the Electrical and Electronics Engineering, Bilkent University, Ankara 06800, Turkey.

Color versions of one or more of the figures in this paper are available online at http://ieeexplore.ieee.org.

Digital Object Identifier 10.1109/JSTQE.2009.2032668 negative effective permeability media [5]-[8]. One can also obtain double-negative medium by using all dielectric media at microwave and optical regimes [9]-[12].

A medium of periodically arranged, subwavelength, highpermittivity ferroelectric rods was demonstrated to have effective negative permeability due to the induced large displacement currents that create magnetic resonance in the presence of the applied electromagnetic field at the $\mathrm{GHz}$ regime [5]. A temperature tunable $\mu$-negative medium, which operates at the first Mie resonance mode of the constituting ferroelectric cubes, was observed between 13.65 and $19.65 \mathrm{GHz}$ [6]. The properties of ferromagnetic materials have led to novel opportunities for $\mu$-negative medium designs. In the vicinity of ferromagnetic resonance (FMR), the effective permeability of ferromagnetic materials can be negative. This phenomenon was demonstrated experimentally by using YIG slabs at $10 \mathrm{GHz}$ [7] and ( $\mathrm{La}: \mathrm{Sr}) \mathrm{MnO}_{3}$ layers at $90 \mathrm{GHz}$ [8]. These are the few examples of metamaterials utilizing ferrosubstances. A rather widespread technique of realizing metamaterials involves nonmagnetic metal-dielectric components.

Electrically small nonmagnetic metallic resonators are proposed as constituting elements of a $\mu$-negative medium [2] and metallic wire mesh structures provide a low-frequency plasma system with negative permittivity [3]. An experimental demonstration of a double-negative $(\varepsilon<0, \mu<0)$ medium (DNG) as a superposition of split ring resonators (SRRs) and wire mesh medium was realized [4] and its unusual properties, such as negative index of refraction, negative phase velocity, reversal of Cherenkov radiation, and Doppler shift have attracted much attention. Applications of DNG and single-negative (SNG) media involve the electromagnetic phenomena of reflection, absorption, radiation, cloaking, refraction, and subwavelength imaging. Planar reflectors that operate like an artificial magnetic conductor (AMC) with high surface impedance have been demonstrated at gigahertz frequencies [13], [14]. A miniaturized rectangular patch antenna with a $\mu$-negative medium substrate operating at $250 \mathrm{MHz}$ [15] as well as an electrically small circular patch antenna loaded with a $\mu$-negative medium are characterized experimentally and theoretically [16]. A negativepermeability-medium-element-loaded monopole antenna was demonstrated experimentally at around $4 \mathrm{GHz}$ in terms of its fundamental limitations [17] and multiple element effects [18]. A negative-permittivity-shell-loaded monopole antenna was developed analytically [19]. In principle, one can enhance the 


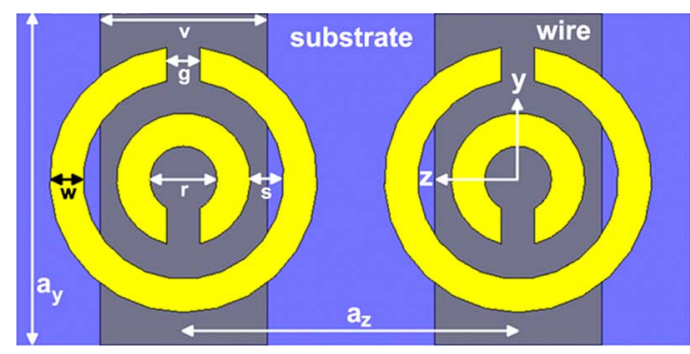

Fig. 1. Parameters of the CMM.

transmission through a subwavelength aperture by utilizing a $\mu$-negative medium cover [20]. This topic was demonstrated theoretically for the periodically arranged subwavelength holes by using deep subwavelength resonators [21] in addition to the previous photonic crystal and surface-grating-aided-enhanced transmission studies [22]-[25]. By using a metamaterial cover it is possible to nearly cloak a subwavelength object at a particular frequency [26]-[28]. The negative refraction property of metamaterials [4], [29] leads to a rather important application: the subwavelength imaging [30].

It is important to demonstrate a $\mu$-negative medium at different regimes of the electromagnetic spectrum. At the megahertz region, $\mu$-negative medium elements lead to the improvement of magnetic resonance imaging by guiding the flux from the object to the receiver [31]. Electrically small metallic elements on planar substrates that operate at the megahertz and gigahertz region and their potential applications have been extensively studied theoretically and experimentally [32]-[34]. E-beam lithography techniques allow us to obtain single-ring SRRs of a 110 $\mathrm{nm}$ side length [35]. By scaling the physical size of the SRRs, a magnetic response at around 5 [36], 6 [37], 100 [38], and $370 \mathrm{THz}$ [39] were demonstrated experimentally. On the other hand, it has been extensively studied that on the process of size scaling due to the ohmic losses of metallic features, the magnetic resonance of SRRs saturate as the operation frequency increases [35], [40], [41]. One way to overcome this problem was to introduce more splits [42], [43] or by using cut wire pairs [44], and thereby, reducing the total resonator capacitance. The resonant frequency increased via this method, but the physical size remained the same. Therefore, the electrical size of the element increased and became comparable to the operation wavelength. The realization of an effective medium becomes a problematic issue as we increase the electrical size of the constituting elements. Another method might be to decrease the environment temperature to enhance the negative permeability [45]. In the present work, we analyzed an SRR-based metamaterial medium operating at the millimeter-wave regime.

\section{DESIGN AND EXPERIMENT}

Our design was realized by utilizing the well-developed printed circuit board (PCB) technology. Fig. 1 shows the schematic view of the manufactured metamaterial layers. On the front face, we have the SRRs with parameters: width of the strips, $w=55 \mu \mathrm{m}$, separation between the adjacent strips, $s$ $=55 \mu \mathrm{m}$, split width, $g=55 \mu \mathrm{m}$, and inner circle diameter, $r=110 \mu \mathrm{m}$. The period in the $z$ - and $y$-direction are $a_{z}=$ $a_{y}=550 \mu \mathrm{m}$. In terms of free space wavelength the period at the propagation direction $\left(a_{z}\right)$ and one of the lateral directions $\left(a_{y}\right)$ was approximately equal to $\lambda_{0} / 6$ at around the resonance frequency. The substrate was Rogers RT/duroid 5880 with relative permittivity, $\varepsilon_{r}=2.0$ and loss tangent, $\tan \delta=0.0009$. The thickness of the substrate was $250 \mu \mathrm{m}$ and the deposited copper thickness was $9 \mu \mathrm{m}$. On the back face of the PCB, we had continuous wires strips at a $v=275 \mu \mathrm{m}$ width. The copper pattern tolerance values $(55 \mu \mathrm{m})$ were nearly at the edge of the current PCB technology. For the slab characterization simulations we used a linearly polarized plane wave propagating at the $-z$-direction is incident upon the metamaterial layers, the $E$-field is at the $y$-direction and the $H$-field is at the $x$-direction. In our simulations, we used the commercial full-wave code, CST Microwave Studio, which is based on the finite integration technique [46]. The unit cell of the metamaterial medium under test is inserted into the simulation domain with periodic boundary conditions at the lateral directions ( $y$ - and $x$-directions). We simulated the reflected and transmitted time signals obtained the relevant complex $S$-parameters.

The experiments were performed by using a millimeter-wave network analyzer with a $50 \mathrm{~dB}$ dynamic range from 75 to 115 GHz. The transmission data in air were obtained by the aid of two standard gain horn antennae. The orientation of and distance between the antennae were kept fixed during the experiments. The media have $30 \times 45$ number of unit cells along the $x$ - and $y$-directions, and the number of unit cells along the propagation direction was varied as $3,5,7$, and 9 . The space between the metamaterial layers at the $x$-direction was $254 \mu \mathrm{m}$. We inserted the metamaterial slab between the horn antennae and measured its transmission response, and then removed the slab and noted the calibration data. By measuring the transmission response of the four different media, we characterized the composite metamaterial medium (CMM) based on the qualitative effective medium theory [47], [48].

\section{TRansmission-Based Qualitative EfFective Medium THEORY ANALYSIS}

In the effective medium theory analysis, we considered the transmission response of four different unit cell structures and concluded whether the CMM was double negative. The SRR and closed-ring resonator (CRR) geometry and corresponding induced surface current at the resonance frequency are all shown in Fig. 2(a) and (b). Mostly, due to the distributed capacitance between the two rings of the SRR, we observed induced circulating currents and a magnetic dipole-like response. By shorting the gaps of the SRR, we introduced the CRR structure on which the circulating currents disappear and at the operation frequency CRR acts like an electric dipole. Fig. 3 shows the transmission spectra of the SRR and CRR media. There were three unit cells at the propagation direction. The media were transparent up to $75 \mathrm{GHz}$ (not shown) and a stopband for the SRR-only medium was observed. This gap was not present at the CRR transmission data, indicating its magnetic origin. Next, we considered the $\varepsilon$-negative wire-mesh medium. Its plasma frequency was 


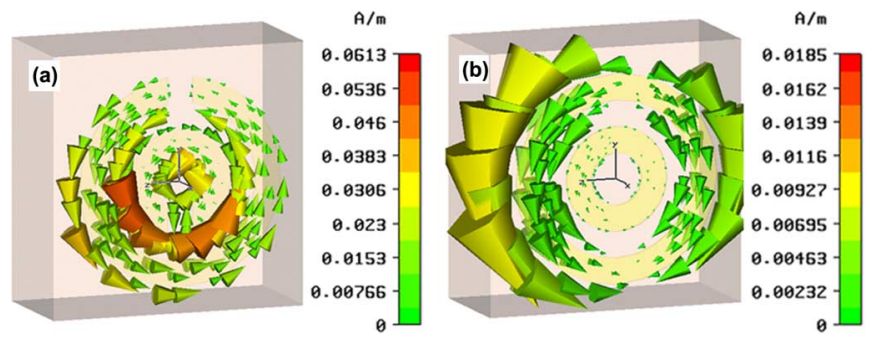

Fig. 2. Schematic view and surface current (a) SRR and (b) shorted SRR, i.e., CRR.

designed to be at around $200 \mathrm{GHz}$. Even the wire-only medium might have negative- $\varepsilon$ below the plasma frequency, thus the composite metamaterial acts as a different plasmonic system. The SRRs of the composite metamaterial kick in the effective permittivity and cause a downward shift of the wire-only medium plasma frequency. Therefore, instead of just considering the wire-only medium transmission response, we should also take into account the transmission response of the CRR and wire composite (closed composite metamaterial, CCMM). We designed the medium parameters in such a way that the plasma frequency of the CCMM was around $150 \mathrm{GHz}$. We can guarantee thereby that the CMM medium is $\varepsilon$-negative below 150 GHz. Finally, we concluded that the transmission peak at around $100 \mathrm{GHz}$, as shown in Fig. 3, was due to the double-negative nature of the CMM medium. Our simulation and experimental results are in good agreement, and we would like to emphasize that the experiment data are the average of many reproducible measurements. As expected, the transmission peak value $\sim-2.5$ $\mathrm{dB}$ was lower than the ideal simulation result. On the other hand, by using a low-loss substrate and rather thick metal coating, we improved the $\sim-25 \mathrm{~dB}$ transmission peak value of our group's previous $100 \mathrm{GHz}$ metamaterial demonstration [49]. Moreover, instead of photolithography techniques, we achieved the desired double-negative properties at the millimeter-wave regime by using a rather cheap technology. At the production step we stacked 30 planar layers of the medium under test in order to cover the entire incident beam. For the SRR-based metamaterial medium, the transmission response was not very sensitive to the angle between the antenna emission direction and metamaterial slab normal [50], or to the small misalignment of the layers [51]. This was not the case for the planar metamaterial media such as the fishnet structure [52].

\section{RETRIEVAl ANALYSIS}

Another well-developed characterization method of metamaterials is the standard retrieval procedure [53]-[55]. The assigned effective refractive index $(n)$ and relative impedance $(z)$ values of the metamaterial slab can be extracted by using the complex scattering parameters with respect to $50 \Omega$. If the metamaterial slab under test is symmetric with respect to the $x-y$ plane, i.e., $S_{11}=S_{22}$ and $S_{21}=S_{12}$, we can extract the effective medium parameters $(n, z)$ of the metamaterial by using the terminology that was developed for the homogenous slab. By using $\varepsilon=n z$, and $\mu=n / z$ formula, we derive the effective per-

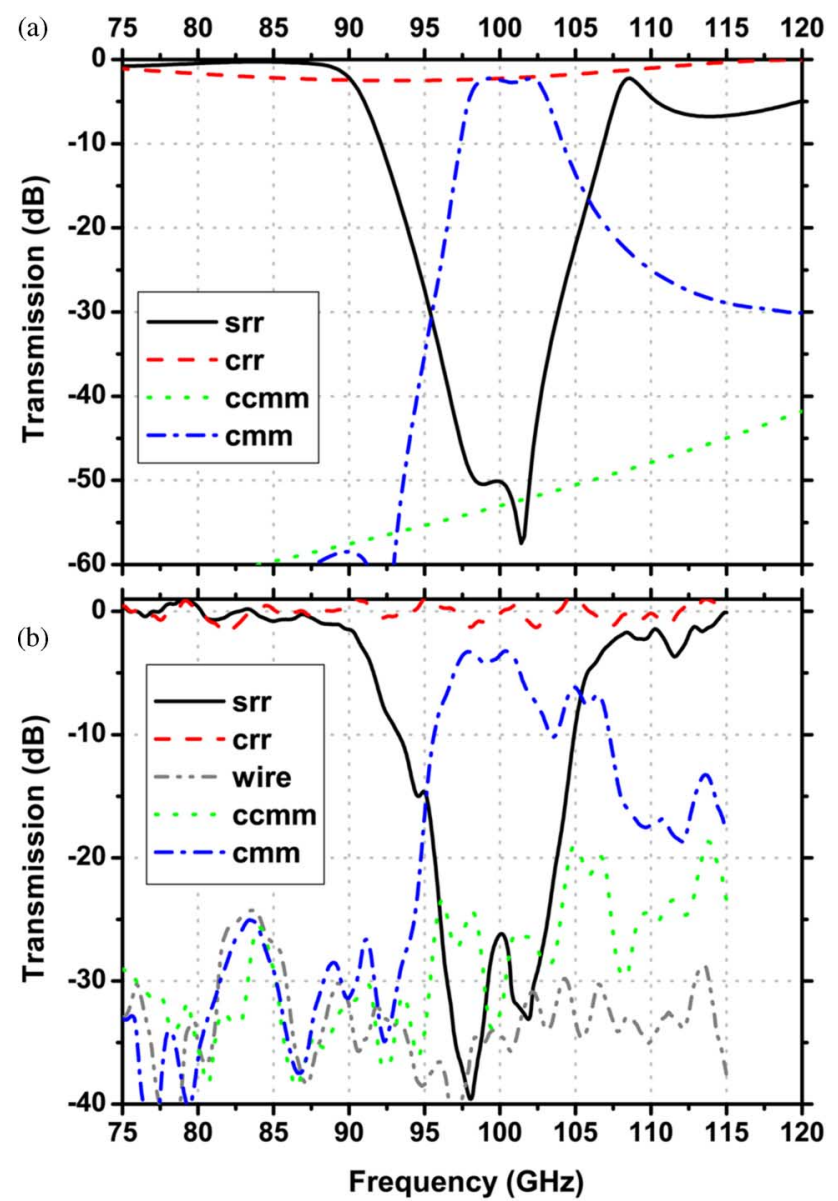

Fig. 3. Transmission spectrum for three-layered metamaterials in the propagation direction. Response of the SRR, CRR, CMM, and shorted CMM, i.e., CCMM are shown. (a) Simulation. (b) Experiment.

mittivity and permeability of the media. The complex scattering parameters for the case of our metamaterial were obtained from the simulations and by using the formalism given in [53]-[55]. We extracted the $n, z, \varepsilon$, and $\mu$ parameters of the CMM medium and are shown in Fig. 4. It is clear that at around $100 \mathrm{GHz}$, the CMM medium is double negative.

At this point, we would like to emphasize that just by itself the passband region shown in the qualitative effective medium theory cannot be claimed to be double negative. Either the complete transmission analysis of the four structures should be studied or the passband region should be supported by the retrieval analysis. Here, we demonstrated the correlation between the two independent well-developed effective medium theory analyses: the retrieval and transmission-based effective medium theory.

\section{LOSS AND BANDWIDTH ANALYSIS}

The resonant nature leads the narrow bandwidth of metamaterials. We calculate the fractional bandwidth of the negative region via $\mathrm{FBW}=\Delta f l f_{0}$, where $\Delta f$ is the half-power bandwidth and $f_{0}$ is the center frequency. We obtained for the three-layer case $\Delta f=4.8 \mathrm{GHz}, f_{0}=99.9 \mathrm{GHz}$, and $\mathrm{FBW}=4.8 \%$.

Fig. 5 shows the effect of increasing the number of layers at the propagation direction to the transmission response. As 


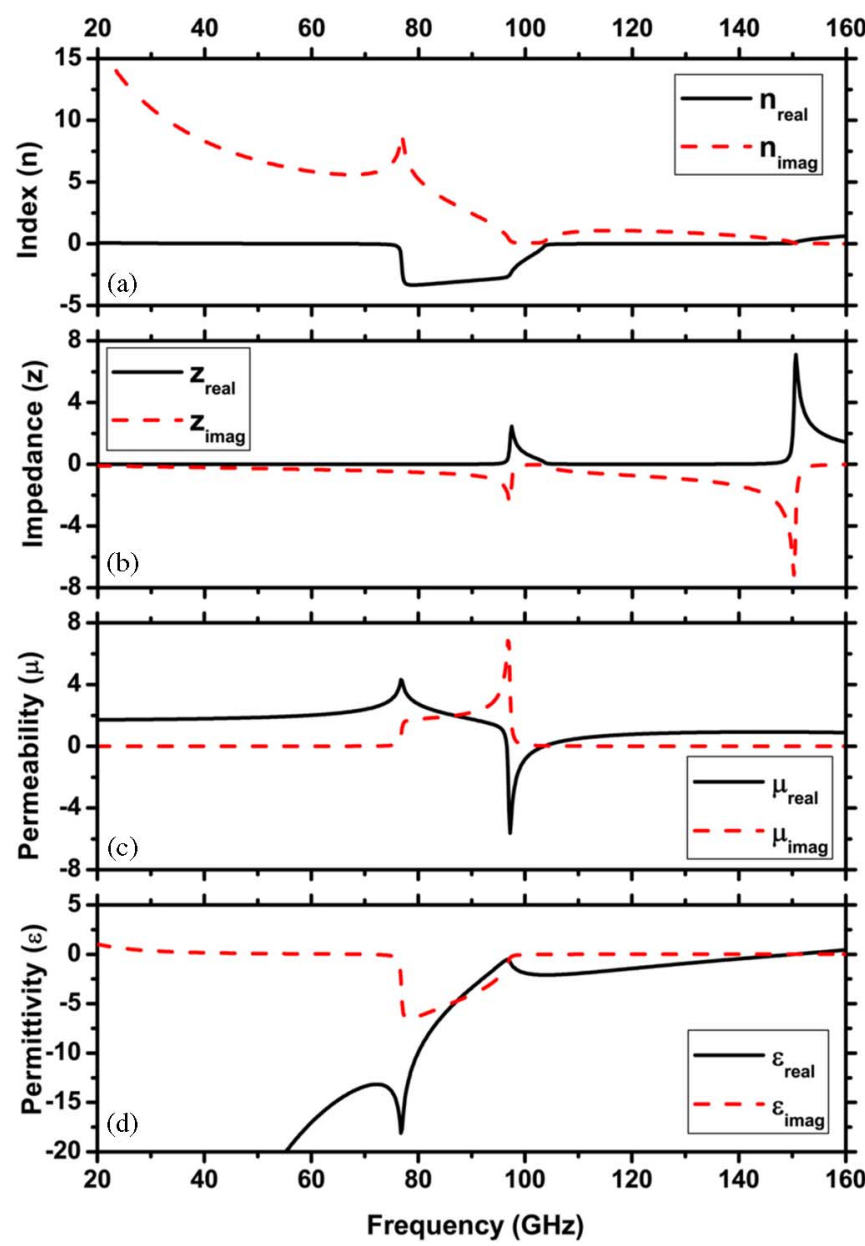

Fig. 4. Extracted parameters as a function of frequency for the SRR-based metamaterial medium. (a) Refractive index. (b) Impedance. (c) Permeability. (d) Permittivity.

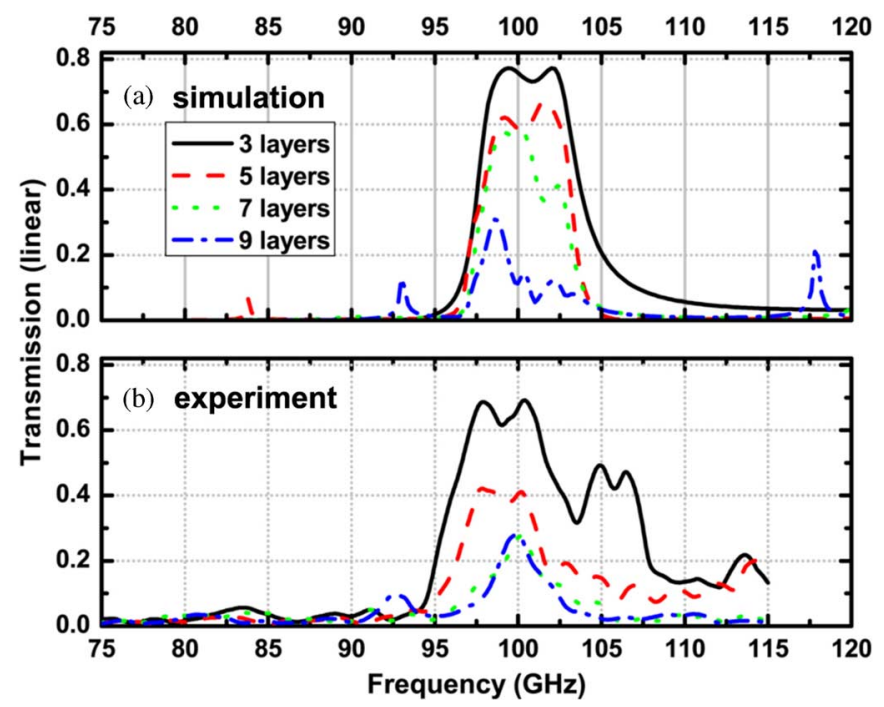

Fig. 5. Transmission spectra in the linear scale for a several number of CMM layers in the propagation direction. (a) Simulations. (b) Experiments.
TABLE I

CALCULATEd LosS AND FBW PARAMETERS FOR THE INCREASED NUMBER OF METAMATERIAL LAYERS IN THE PROPAGATION DiRECTION

\begin{tabular}{|c|c|c|c|c|}
\hline Number of Layers & 3 & 5 & 7 & 9 \\
\hline Loss $(\mathrm{dB} / \mathrm{mm})$ & 2.1 & 2.8 & 2.9 & 2.2 \\
\hline FBW $(\%)$ & 7.5 & 5 & 3.9 & 3.3 \\
\hline
\end{tabular}

expected, the total loss (in decibels per millimeter) increases as the number of layers increase. We summarized the resulting loss and bandwidth data at $100 \mathrm{GHz}$ in Table I. We can clearly state that as we increase the number of metamaterial layers at the propagation direction, the total loss increases and the FBW decreases.

\section{FAR-FIELD RADIATION BEHAVIOR OF HORN METAMATERIAL COMPOSITE}

Fig. 6 displays measured and calculated far-field pattern of the metamaterial lens/horn antenna composite structure. We have modeled the homogeneous metamaterial by using DrudeLorentz model in a way that at the frequency of interest the retrieved $\varepsilon$ and $\mu$ values almost coincide. Other parameters in the simulations are kept the same as experiment. At the farfield, $R=38 \mathrm{~mm}$, by implementing an automated experiment setup, we scanned the field intensity for both the horn antenna and for the hybrid structure. Being in good agreement the simulation, the experimental results showed that the metamaterial lens decreases the angular width of the hybrid structure's radiation pattern at the E-plane. Due to the experimental limitations, we could not be able to scan the less interesting H-plane and could not comment on the directivity, gain, and efficiency of the hybrid structure. We observed that the maximum transmitted peak value was $2-3 \mathrm{~dB}$ less than the horn-only case due to the lossy nature of the metamaterial lens. The behavior of the hybrid structure composed of a horn antenna as driven element and DNG material as director can be considered as a novel phenomenon, which is similar to the behavior of Yagi-Uda antenna. The DNG part is passive and thin. It forms a rough image of the source. Its rough image and source itself can be used to explain the higher directivity of the composite system. In our millimeter-wave setup, we could only use horn antennas as the source and sink. Thereby, we have not tried to observe or optimize this effect with different sources. Moreover, the number of DNG materials at the propagation direction could not be increased due to our fabrication and measurement limitations. On the other hand, Fig. 6(d) shows the numerical result for the case of two DNG materials at the propagation direction. We showed that the directivity further increased in this case. The separation between the DNG materials was equal to their thicknesses, and neither the thicknesses nor the separation between them was optimized. Here, we prefer to qualitatively demonstrate and explain the observed phenomenon.

\section{Study OF A Metamaterial PRISM}

We synthesized a prism-shaped metamaterial that was composed of stacked seven-layered blocks, in the lateral (x) direction. There were six different block types with unit cells in the 


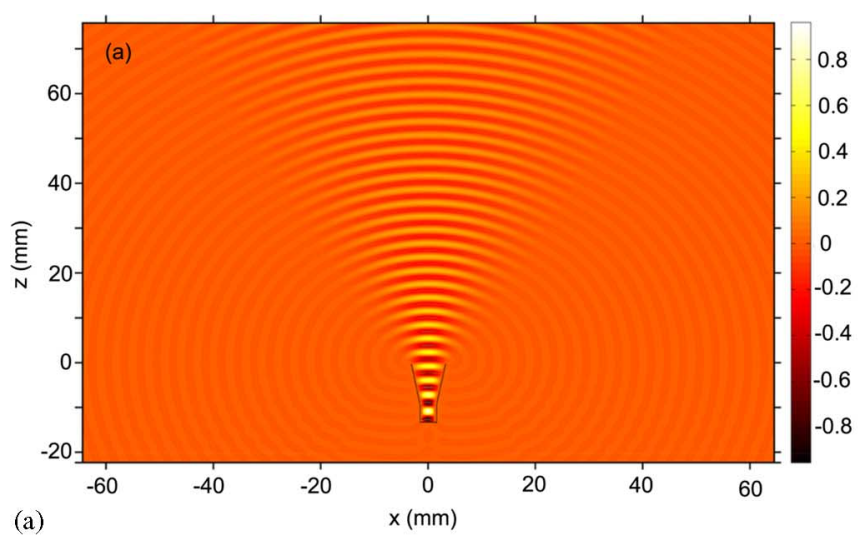

(a)
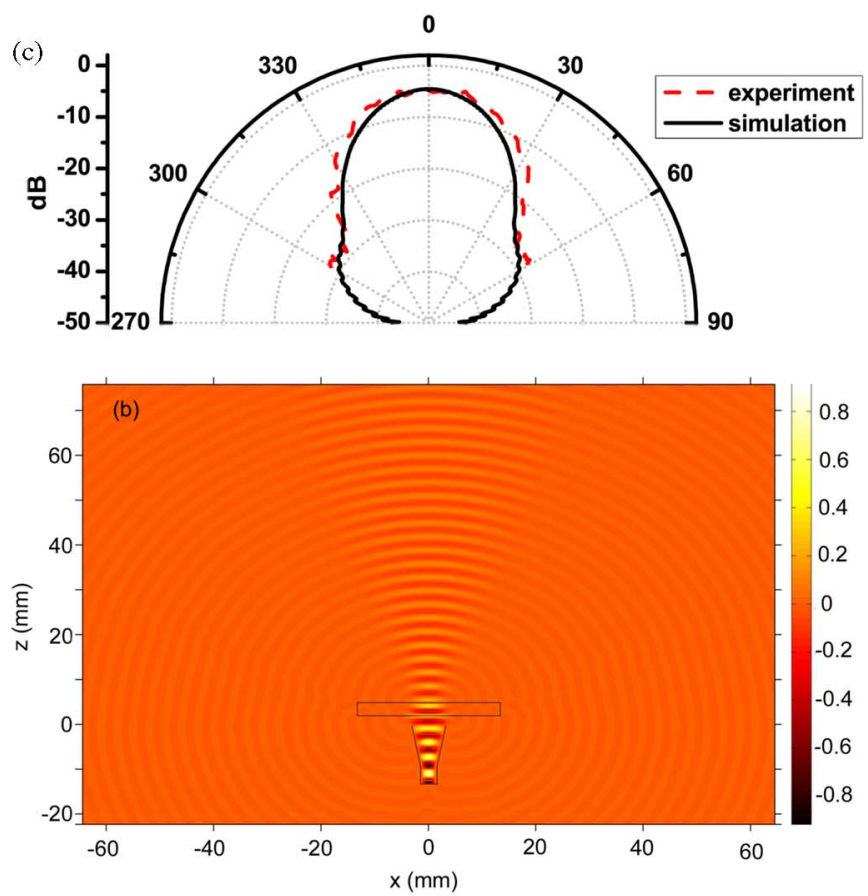

(d)

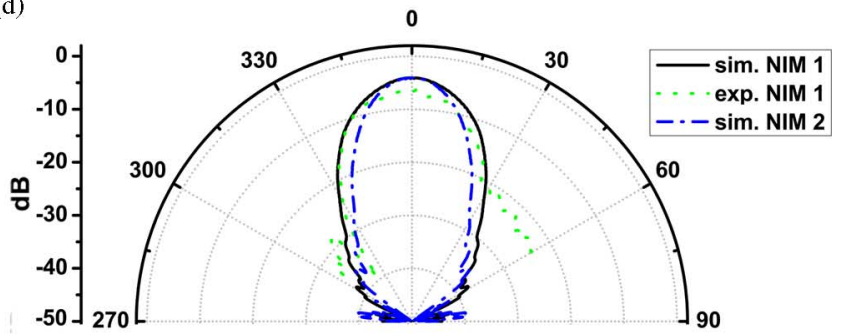

Fig. 6. Simulated field map of (a) horn antenna, (c) horn antenna and metamaterial lens (hybrid structure) at $99 \mathrm{GHz}$. Focusing and redistribution of waves can be seen in (b). Far-field patterns of (b) horn antenna and (d) hybrid structures with 1 and 2 negative index metamaterial (NIM) slabs at the propagation direction.

propagation direction ranging from 3 to 9, as shown in Fig. 7. The angle between the prism's second interface and propagation vector was $\alpha=8.4^{\circ}$. The setup consisted of a millimeter-wave network analyzer, an automated rotary scanner system, and two horn antennas as the transmitter and receiver. The transmitter horn antenna was parallel to the first metaprism interface. We scanned the angular transmission intensity at a distance of

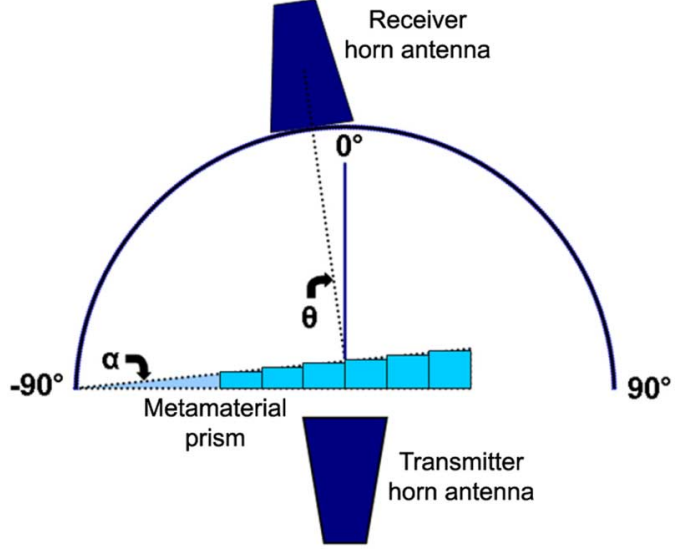

Fig. 7. Schematic of the setup used in the millimeter-wave metamaterial prism experiment. The metamaterial sample, source and detector antennas, and airprism second interface normal are shown. The prism angle $\alpha=8.4^{\circ}$ and scanning angle $\theta$ were changed from $-60^{\circ}$ to $60^{\circ}$.

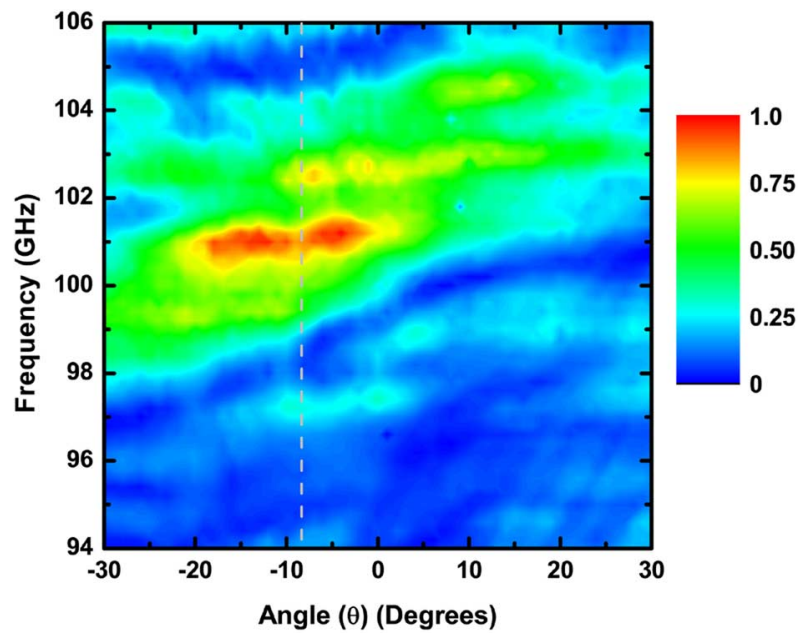

Fig. 8. Transmission spectra as a function of the frequency and scanning angle $\theta$.

$R=38 \mathrm{~mm}$, which corresponded to the far-field of the antennas from 75 to $115 \mathrm{GHz}$. Fig. 8 shows the transmission spectra as a function of the frequency and scanning angle. As the frequency increases, the beam refracts from the negative to positive side. The transition can be seen from a negative index to positive index in Fig. 8. We would like to point out the frequency band at which the beam was refracted to the negative side coincides with double-negative region predicted by the effective medium analyses. The unit cells of the metamaterial under study were adequately electrically small so that the periodicity related effects were minor.

Fig. 9 shows the frequency cuts that were taken from the experimental data at $100 \mathrm{GHz}$. The normal of the second interface was demonstrated with a dashed line and arrow. We can compare the results of the free space and metaprism cases in this figure. A negatively refracted beam emerges on the lefthand side of the prism normal, whereas the beam emerged on the right-hand side for the positive index medium. We also confirmed the experimental results by using CST simulations, 


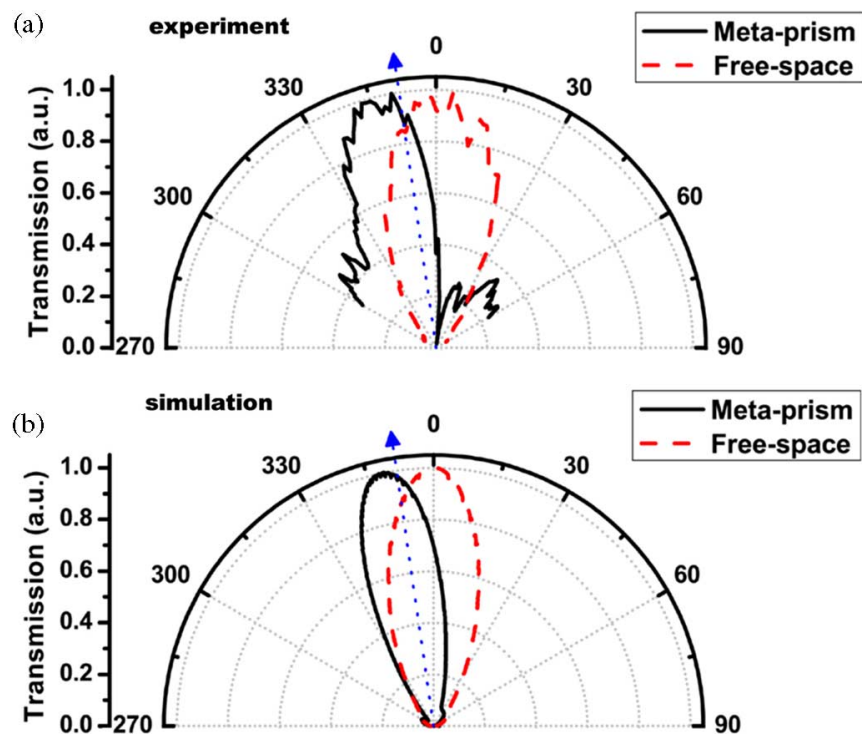

Fig. 9. Frequency cuts of the transmission spectra at $100 \mathrm{GHz}$ for the free space and metaprism. (a) Experiments. (b) Simulations.

in which the negative index prism was modeled with the Drude-Lorentz dispersion theory. The Drude-model parameters were as follows: the plasma frequency was equal to, $\omega_{p}=$ $2 \cdot \pi \cdot 130 \mathrm{GHz}$, the collision frequency was equal to, $v_{c}=$ $0.01 \mathrm{~Hz}$. For the magnetic dispersion, the following Lorentzmodel parameters were used: permeability at infinity was equal to, $\mu_{\infty}=1$, permeability static was $\mu_{s}=1.07$, resonance frequency was $\omega_{\text {res }}=2 \cdot \pi \cdot 97.9 \mathrm{GHz}$, and the damping frequency was $\gamma=1.3 \mathrm{GHz}$. The retrieved parameters of the metamaterial medium were taken into account when determining the Drude-Lorentz model parameters. At around the frequency of interest, the retrieved $\varepsilon$ and $\mu$ values were as close as possible to the Drude-Lorentz values. In this simulation, we applied the perfect electric conductor boundary conditions at the vertical direction $(y)$ and in the other directions the perfect absorbing boundary conditions were applied. The physical parameters of the transmitter horn antenna used in the simulations were the same as the experimental configuration except a waveguide port was placed instead of a coaxial probe feed. By this method, we obtained the field propagation and far-field profiles with affordable computation power in a reasonable amount of time. Fig. 10 shows the field profile. The negatively refracted beam emerging from the second prism interface, the negative phase velocity inside the metamaterial medium, as well as the reflection properties can be seen in this figure and corresponding animation.

In this paper, we conveyed the $S$-band studies of the metamaterials to the millimeter-wave regime and demonstrated the feasibility of rather low-loss metamaterial-based devices in this regime. We experimentally demonstrated the prism effect and radiation properties of an antenna composed of the metamaterial slab and a standard horn antenna. For our group's previously demonstrated $100 \mathrm{GHz}$ paper [49], the losses were much higher and experiments, such as negative index prism, oblique incidence negative refraction were much difficult to perform. Here, we demonstrated the usability of a classical design approach at

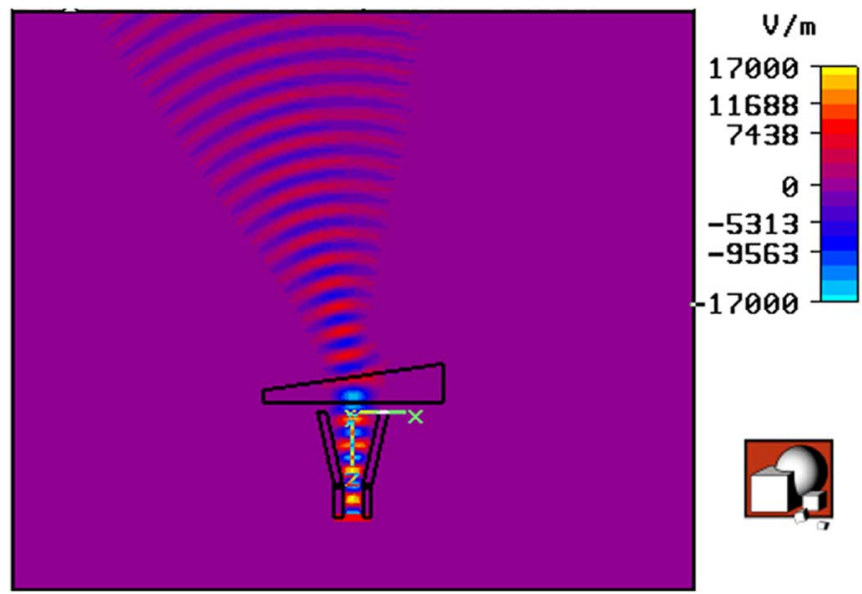

Fig. 10. 2-D map of the electric field amplitude at the $y$-direction. Negative refraction and negative phase velocity can be seen (media 1: size $1.00 \mathrm{MB}$, format MPEG).

millimeter-wave frequencies, at which the ordinary PCB technology has serious limitations. The utilized simulation technique allowed us to observe the field distribution in double-negative metamaterial-based devices operating at the millimeter-wave regime with a rather low computational power. The fabricated elements had $\sim 50 \mu \mathrm{m}$ minimum linewidths and half-millimeter unit cells. The fabrication and handling of these structures were rather difficult compared to the ones operating at the $S$-band and $X$-band.

\section{CONCLUSION}

In summary, the characterization of SRR-based metamaterial operating at $100 \mathrm{GHz}$ was demonstrated in terms of the transmission-based qualitative effective medium theory and the standard retrieval analysis. The structure layers were produced via PCB technology and the transmission response for increasing the number of layers at the propagation direction was analyzed. We observed a stopband for the SRR-only medium and passband for the CMM medium at around $100 \mathrm{GHz}$. The transmission peak value was $\sim-2.5 \mathrm{~dB}$ and the metamaterials' average loss was $\sim 2.5 \mathrm{~dB} / \mathrm{mm}$. The experimental results were not very sensitive to the layer disorders or the angle of incidence, and they were in good agreement with the numerical calculations. The far-field radiation response of horn antenna and metamaterial slab composite had a lower angular width, which was verified both numerically and experimentally. Finally, by constructing a metamaterial prism and performing angular scan experiments we confirmed the retrieved negative index property of a SRR-based metamaterial.

\section{REFERENCES}

[1] N. W. Ashcroft and N. D. Mermin, Eds., Solid State Physics. Fort Worth, TX: Saunders College, 1976.

[2] J. B. Pendry, A. J. Holden, D. J. Robbins, and W. J. Stewart, "Magnetism from conductors and enhanced nonlinear phenomena," IEEE Trans. Microw. Theory Tech., vol. 47, no. 11, pp. 2075-2084, Aug. 1999.

[3] J. B. Pendry, A. J. Holden, W. J. Stewart, and I. Youngs, "Extremely low frequency plasmons in metallic mesostructures," Phys. Rev. Lett., vol. 76 , pp. 4773-4776, 1996. 
[4] R. A. Shelby, D. R. Smith, and S. Schultz, "Experimental verification of a negative index of refraction," Science, vol. 292, pp. 77-79, 2001.

[5] L. Peng, L. Ran, H. Chen, H. Zhang, J. A. Kong, and T. M. Grzegorczyk, "Experimental observation of left-handed behavior in an array of standard dielectric resonators," Phys. Rev. Lett., vol. 98, pp. 157403-157401157403-157404, 2007.

[6] Q. Zhao, B. Du, L. Kang, H. Zhao, Q. Xie, B. Li, X. Zhang, J. Zhou, L. Li, and Y. Meng, "Tunable negative permeability in an isotropic dielectric composite," Appl. Phys. Lett., vol. 92, pp. 051106-051101-051106051103, 2008.

[7] H. Zhao, J. Zhou, Q. Zhao, B. Li, L. Kang, and Y. Bai, "Magnetotunable left-handed material consisting of yttrium iron garnet slab and metallic wires," Appl. Phys. Lett., vol. 91, pp. 131107-131101-131107-131103, 2007.

[8] A. Pimenov, A. Loidl, P. Przyslupski, and B. Dabrowski, "Negative refraction in ferromagnet-superconductor superlattices," Phys. Rev. Lett., vol. 95, pp. 247009-247001-247009-247004, 2005.

[9] O. G. Vendik and M. S. Gashinova, "Artificial double negative (dng) media composed by two different sphere lattices embedded in a dielectric matrix," in Proc. 34th Eur. Microw. Conf., Amsterdam, The Netherlands, 2004, pp. 1209-1212.

[10] A. Ahmadi and H. Mosallaei, "Physical configuration and performance modeling of all-dielectric metamaterials," Phys. Rev. B, vol. 77, pp. 045104-045101-045104-045111, 2008

[11] C. L. Holloway, E. F. Kuester, J. Baker-Jarvis, and P. Kabos, “A double negative (dng) composite medium composed of magnetodielectric spherical particles embedded in a matrix," IEEE Trans. Antennas Propag., vol. 51, no. 10, pp. 2596-2603, Oct. 2003.

[12] S. Ghadarghadr and H. Mosallaei, "Dispersion diagram characteristics of periodic array of dielectric and magnetic materials based spheres," IEEE Trans. Antennas Propag., vol. 57, no. 1, pp. 149-160, Jan. 2009.

[13] D. Sievenpiper, L. Zhang, R. F. J. Broas, N. G. Alexopolous, and E. Yablonovitch, "High-impedance electromagnetic surfaces with a forbidden frequency band," IEEE Trans. Microw. Theory Tech., vol. 47, no. 11 , pp. 2059-2074, Nov. 1999.

[14] A. Ourir, A. Lustrac, and J. M. Lourtioz, "All-metamaterial-based subwavelength cavities $(\lambda / 60)$ for ultrathin directive antennas," Appl. Phys. Lett., vol. 88, pp. 084103-084101-084103-084103, 2006.

[15] K. Buell, H. Mosallaei, and K. Sarabandi, "A substrate for small patch antennas providing tunable miniaturization factors," IEEE Trans. Microw. Theory Tech., vol. 54, no. 1, pp. 135-146, Jan. 2006.

[16] A. Alu, F. Bilotti, N. Engheta, and L. Vegni, "Subwavelength compact resonant patch antennas loaded with metamaterials," IEEE Trans. Antennas Propag., vol. 55, no. 1, pp. 13-25, Jan. 2007.

[17] K. B. Alici and E. Ozbay, "Electrically small split ring resonator antennas," J. Appl. Phys., vol. 101, pp. 083104-083101-083104-083104, 2007.

[18] K. B. Alici and E. Ozbay, "Radiation properties of a split ring resonator and monopole composite," Phys. Solidi Status B, vol. 244, pp. 1192-1196, 2007.

[19] A. Erentok and R. Ziolkowski, "A hybrid optimization method to analyze metamaterial-based electrically small antennas," IEEE Trans. Antennas Propag., vol. 55, no. 3, pp. 731-741, Mar. 2007.

[20] A. Alu, F. Bilotti, N. Engheta, and L. Vegni, "Metamaterial covers over a small aperture," IEEE Trans. Antennas Propag., vol. 54, no. 6, pp. 16321643, Jun. 2006

[21] K. B. Alici, F. Bilotti, L. Vegni, and E. Ozbay, "Optimization and tunability of deep subwavelength resonators for metamaterial applications: Complete enhanced transmission through a subwavelength aperture," Opt. Exp., vol. 17, pp. 5933-5943, 2009.

[22] I. Bulu, H. Caglayan, and E. Ozbay, "Beaming of light and enhanced transmission via surface modes of photonic crystals," Opt. Lett., vol. 30, pp. 3078-3080, 2005 .

[23] S. S. Akarca-Biyikli, I. Bulu, and E. Ozbay, "Enhanced transmission of microwave radiation in one-dimensional metallic gratings with sub-wavelength aperture," Appl. Phys. Lett., vol. 85, pp. 1098-1100, 2004.

[24] I. Bulu, H. Caglayan, and E. Ozbay, "Highly directive radiation from sources embedded inside photonic crystals," Appl. Phys. Lett., vol. 83, pp. 3263-3265, 2003.

[25] H. Caglayan, I. Bulu, and E. Ozbay, "Extraordinary grating-coupled microwave transmission through a subwavelength annular aperture," Opt. Exp., vol. 13, pp. 1666-1668, 2005.

[26] J. B. Pendry, D. Schurig, and D. R. Smith, "Controlling electromagnetic fields," Science, vol. 312, pp. 1780-1782, 2006.
[27] A. Alu and N. Engheta, "Plasmonic materials in transparency and cloaking problems: Mechanism, robustness, and physical insights," Opt. Exp., vol. 15, pp. 3318-3332, 2007.

[28] S. Guenneau, S. A. Ramakrishna, S. Enoch, S. Chakrabarti, G. Tayeb, and B. Gralak, "Cloaking and imaging effects in plasmonic checkerboards of negative $\varepsilon$ and $\mu$ and dielectric photonic crystal checkerboards," Photon. Nanostruct., vol. 5, pp. 63-72, 2007.

[29] C. G. Parazzoli, R. B. Greegor, K. Li, B. E. C. Koltenbah, and M. Tanielian, "Experimental verification and simulation of negative index of refraction using Snell's law," Phys. Rev. Lett., vol. 90, pp. 107401-107401-107401107404, 2003.

[30] K. Aydin, I. Bulu, and E. Ozbay, "Subwavelength resolution with a negative-index metamaterial superlens," Appl. Phys. Lett., vol. 90, pp. 254102-254101-254102-254104, 2007.

[31] M. C. K. Wiltshire, J. B. Pendry, I. R. Young, D. J. Larkman, D. J. Gilderdale, and J. V. Hajnal, "Microstructured magnetic materials for rf flux guides in magnetic resonance imaging," Science, vol. 291, pp. 849-851, 2001.

[32] F. Bilotti, A. Toscano, and L. Vegni, "Design of spiral and multiple splitring resonators for the realization of miniaturized metamaterial samples," IEEE Trans. Antennas Propag., vol. 55, no. 8, pp. 2258-2267, Aug. 2007.

[33] K. B. Alici, F. Bilotti, L. Vegni, and E. Ozbay, "Miniaturized negative permeability materials," Appl. Phys. Lett., vol. 91, pp. 071121-071121071121-071123, 2007

[34] L. Zhang, G. Tuttle, and C. M. Soukoulis, "Ghz magnetic response of split ring resonators," Photon. Nanostruct., vol. 2, pp. 155-159, 2004.

[35] M. W. Klein, C. Enkrich, and M. Wegener, "Single-slit split-ring resonators at optical frequencies: Limits of size scaling," Opt. Lett., vol. 31 , pp. 1259-1261, 2006.

[36] B. D. F. Casse, M. O. Moser, J. W. Lee, M. Bahou, S. Inglis, and L. K. Jian, "Towards three-dimensional and multilayer rod-split-ring metamaterial structures by means of deep x-ray lithography," Appl. Phys. Lett., vol. 90, pp. 254106-254101-254106-254103, 2007.

[37] N. Katsarakis, G. Konstantinidis, A. Kostopoulos, R. S. Penciu, T. F. Gundogdu, M. Kafesaki, E. N. Econoumou, Th. Koschny, and C. M. Soukoulis, "Magnetic response of split-ring resonators in the far-infrared frequency regime," Opt. Lett., vol. 30, pp. 1348-1351, 2005.

[38] S. Linden, C. Enkrich, M. Wegener, J. Zhou, Th. Koschny, and C. M. Soukoulis, "Magnetic response of metamaterials at 100 terahertz," Science, vol. 306, pp. 1351-1353, 2004

[39] C. Enkrich, M. Wegener, S. Linden, S. Burger, L. Zschiedrich, F. Schmidt, J. F. Zhou, Th. Koschny, and C. M. Soukoulis, "Magnetic metamaterials at telecommunication and visible frequencies," Phys. Rev. Lett., vol. 95, pp. 203901-203901-203901-203904, 2005.

[40] A. Ishikawa, T. Tanaka, and S. Kawata, "Negative magnetic permeability in the visible light region," Phys. Rev. Lett., vol. 95, pp. 237401-237401237401-237404, 2005.

[41] J. Zhou, Th. Koschny, M. Kafesaki, E. N. Economou, J. B. Pendry, and C. M. Soukoulis, "Saturation of the magnetic response of split-ring resonators at optical frequencies," Phys. Rev. Lett., vol. 95, pp. $223902-$ 223901-223902-223904, 2005.

[42] S. O'Brien, D. McPeake, S. A. Ramakrishna, and J. B. Pendry, "Nearinfrared photonic band gaps and nonlinear effects in negative magnetic metamaterials," Phys. Rev. B, vol. 69, pp. 241101-241101-241101241104, 2004.

[43] K. Aydin, K. Guven, M. Kafesaki, C. M. Soukoulis, and E. Ozbay, "Investigation of magnetic resonances for different split-ring resonator parameters and designs," New J. Phys., vol. 7, pp. 168-161-168-115, 2005.

[44] A. N. Grigorenko, A. K. Geim, H. F. Gleeson, Y. Zhang, A. A. Firsov, I. Y. Khrushchev, and J. Petrovic, "Nanofabricated media with negative permeability at visible frequencies," Nature, vol. 438, pp. 17-20, 2005 .

[45] K. B. Alici and E. Ozbay, "Low-temperature behavior of magnetic metamaterial elements," New J. Phys., vol. 11, pp. 043015-043011-004015043018, 2009.

[46] CST-Microwave Studio 9.0 CST of America. [Online]. Available: http://www.cst.com

[47] E. Ozbay, K. Aydin, E. Cubukcu, and M. Bayindir, "Transmission and reflection properties of composite double negative metamaterials in free space," IEEE Trans. Antennas Propag., vol. 51, no. 10, pp. 2592-2595, Oct. 2003.

[48] Th. Koschny, M. Kafesaki, E. N. Economou, and C. M. Soukoulis, "Effective medium theory of lefthanded materials," Phys. Rev. Lett., vol. 93, pp. 107402-107401-107402-107404, 2004. 
[49] M. Gokkavas, K. Guven, I. Bulu, K. Aydin, R. S. Penciu, M. Kafesaki, C. M. Soukoulis, and E. Ozbay, "Experimental demonstration of a left-handed metamaterial operating at $100 \mathrm{ghz}$," Phys. Rev. B, vol. 73, pp. 193103-193101-193103-193104, 2006.

[50] K. B. Alici and E. Ozbay, "Oblique response of a split-ring resonator based left-handed metamaterial slab," Opt. Lett., vol. 34, pp. 2294-2296, 2009.

[51] K. Aydin, K. Guven, N. Katsarakis, C. M. Soukoulis, and E. Ozbay, "Effect of disorder on magnetic resonance band gap of split-ring resonator structures," Opt. Exp., vol. 12, pp. 5896-5901, 2004.

[52] K. B. Alici and E. Ozbay, "Characterization and tilted response of a fishnet metamaterial operating at $100 \mathrm{ghz}, "$ J. Phys. D: Appl. Phys., vol. 41, pp. 135011-135011-135011-135015, 2008.

[53] D. R. Smith, S. Shultz, P. Markos, and C. M. Soukoulis, "Determination of effective permittivity and permeability of metamaterials from reflection and transmission coefficients," Phys. Rev. B, vol. 65, pp. 195104-195101195104-195105, 2002.

[54] X. Chen, T. M. Grzegorczyk, B. I. Wu, J. Pacheco, and J. A. Kong, "Robust method to retrieve the constitutive effective parameters of metamaterials," Phys. Rev. E, vol. 70, pp. 016608-016601-016608-016607, 2004.

[55] D. R. Smith, D. C. Vier, Th. Koschny, and C. M. Soukoulis, "Electromagnetic parameter retrieval from inhomogeneous metamaterials," Phys. Rev. E, vol. 70, pp. 036617-036611-036617-036611, 2005.

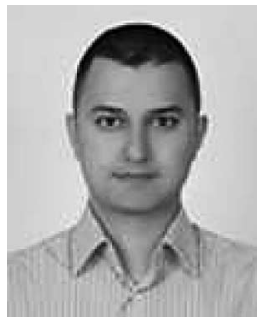

Kamil Boratay Alici was born in Sivas, Turkey, on January 12, 1981. He received the B.S. degree in physics in 2004 from Bilkent University, Ankara, Turkey, where he is currently working toward the Ph.D. degree.

He has authored or coauthored 15 papers in scientific journals. His current research interests include electromagnetic metamaterials: antenna and superlens applications, acoustic metamaterials, and negative refraction in photonic crystals.

Mr. Alici was the recipient of the Undergraduate Scholarship (2001-2004), and the Graduate Scholarship (2004-2009) of the Scientific and Technological Research Council of Turkey (TUBITAK).

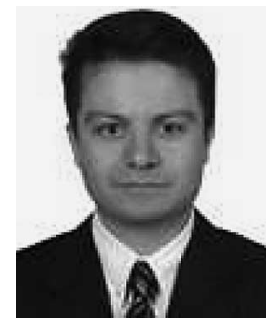

Ekmel Ozbay (M'98) was born in Ankara, Turkey, on March 25, 1966. He received the B.S. degree in electrical engineering from the Middle East Technical University, Ankara, Turkey, in 1983, and the M.S. and Ph.D. degrees in electrical engineering from Stanford University, Stanford, CA, in 1989 and 1992, respectively.

From 1992 to 1994, he was a Scientist with the Department of the Environment (DOE), Ames National Laboratory, Iowa State University, where he was involved in the area of photonic-bandgap materials. In 1995, he joined Bilkent University, Ankara, Turkey, where he is currently a Full Professor in the Department of Electrical and Electronics Engineering and the Department of Physics. He is the Founding Director of the Nanotechnology Research Center, Bilkent University. He has authored or coauthored more than 320 papers in scientific journals, conference proceedings, and books. His current research interests include nanophotonics, metamaterials, metal-organic chemical vapor deposition (MOCVD) growth, and fabrication of GaN-based electronic and photonic devices, photonic crystals, and high-speed optoelectronics. Since 2002, he has been a Topical Editor of Optics Letters.

Dr. Ozbay was the recipient of the 1997 Adolph Lomb Medal of the Optical Society of America and the 2005 European Union Descartes Science Award. 